Cinémas

Revue d'études cinématographiques

Journal of Film Studies

\title{
L'expérience émotionnelle et le style. Le troisième sens, l'excès et le sublime vus à la lumière des états émotifs
}

\section{Johannes Riis}

Volume 12, numéro 2, hiver 2002

Cinéma et cognition

URI : https://id.erudit.org/iderudit/024883ar

DOI : https://doi.org/10.7202/024883ar

Aller au sommaire du numéro

Éditeur(s)

Cinémas

ISSN

1181-6945 (imprimé)

1705-6500 (numérique)

Découvrir la revue

Citer cet article

Riis, J. (2002). L'expérience émotionnelle et le style. Le troisième sens, l'excès et le sublime vus à la lumière des états émotifs. Cinémas, 12(2), 117-134.

https://doi.org/10.7202/024883ar
Résumé de l'article

Dans cet article, la notion de troisième sens de Barthes fournit le point de départ d'une réflexion portant sur un type de réponse spectatorielle qui ne se prête pas en soi à une description en termes de signification, dénotative ou connotative, et qui ne dépend pas de la progression de l'histoire. Dans ce type d'expérience, nous sentons que la situation est significative pour les personnages, mais notre attention est attirée vers des détails apparemment insignifiants, inconnus des personnages puisque ce sont le cadrage et le montage qui les mettent en évidence. En revisitant la notion de style, l'auteur soutient que l'expérience d'un troisième sens ou d'un excès du film peut être considérée comme une expérience du sublime et qu'elle doit être comprise en termes psychologiques, à savoir comme le résultat des processus émotifs et cognitifs du spectateur. 


\section{L'expérience émotionnelle et le style. Le troisième sens, l'excès et le sublime vus à la lumière des états émotifs}

\section{Johannes Riis}

\section{RÉSUMÉ}

Dans cet article, la notion de troisième sens de Barthes fournit le point de départ d'une réflexion portant sur un type de réponse spectatorielle qui ne se prête pas en soi à une description en termes de signification, dénotative ou connotative, et qui ne dépend pas de la progression de l'histoire. Dans ce type d'expérience, nous sentons que la situation est significative pour les personnages, mais notre attention est attirée vers des détails apparemment insignifiants, inconnus des personnages puisque ce sont le cadrage et le montage qui les mettent en évidence. En revisitant la notion de style, l'auteur soutient que l'expérience d'un troisième sens ou d'un excès du film peut être considérée comme une expérience du sublime et qu'elle doit être comprise en termes psychologiques, à savoir comme le résultat des processus émotifs et cognitifs du spectateur.

\section{ABSTRACT}

Barthes'notion of "third meaning" has provided a mean of addressing a kind of spectator response which neither lends itself to description in terms of meaning, denotative or connotative, nor depends on the progression of the story. In this kind of experience, we sense that the situation is significant to the persons photographed, but our attention is drawn to seemingly insignificant details, unknown to the characters since the details are made 
salient by framing or editing. By re-conceptualizing the notion of style, this essay argues that we need to understand the experience of "third meaning" and similar concepts such as "excess" and "sublime" in psychological terms, as the result of cognitive and emotive processes occurring in the spectator.

En 1970 paraissait dans Les Cahiers du cinéma "Le troisième sens. Notes de recherche sur quelques photogrammes de S. M. Eisenstein ». Dans cet essai, Roland Barthes s'attachait à recueillir les effets d'un sens étrange, sens étranger aussi bien à celui de la connotation, ou du sens symbolique, qu'à celui de la dénotation. Ce troisième niveau de sens, celui d'un sens obtus, têtu, échappait à la description, se tenait là où le langage avait cessé, et la linguistique ne serait d'aucun secours pour le saisir. L'essai de Barthes a imprimé sa marque sur les études cinématographiques, qui invitait à considérer les aspects non conceptuels d'un film. Et si c'est aujourd'hui par le terme "excès" que l'on désigne le plus souvent ces aspects, reste que le concept d'excès trouve son point de départ dans la notion barthésienne de troisième sens - c'est le cas notamment dans «The Concept of Cinematic Excess", de Kristin Thompson (1999) ${ }^{1}$. Pour ma part, je retiens de Barthes (1982, p. 51) la conscience qu'il a d'être ému par le contenu des images: le troisième sens «[...] porte une certaine émotion; [...] c'est une émotion-valeur, une évaluation ". Plus précisément, je tenterai de jeter les bases d'une explication quant à l'expérience émotionnelle que procure le film Ivan le Terrible (1944) de Sergei Eisenstein.

Pour ce faire, je considérerai le troisième sens et l'excès puis le sublime - à la lumière de ce que certains psychologues qualifient de «cognitively undefined emotions». Être ému, touché, affecté ou emporté par un film, c’est éprouver des émotions non définies par la cognition. Voilà le genre d'expériences émotionnelles qui m'intéresse. Les seules caractéristiques d'un film ne suffisent pas à rendre compte de telles expériences; il faut aussi s'intéresser à l'activité du spectateur. La présente étude s'inscrit dans une perspective psychocognitive. S'y affirme en effet l'idée qu'il y a une relation fonctionnelle entre le traitement de l'information et les émotions. Il y a plus de quinze ans David 
Bordwell (1985, p. 30) affirmait: "Any theory of the spectator's activity must rest upon a general theory of perception and cognition.» Et des émotions, pouvons-nous ajouter aujourd'hui dans la foulée de travaux récents qui, dans leur diversité, participent de ce qu'on appelle la critique cognitive ${ }^{2}$. En se basant sur les découvertes et les modèles théoriques de la psychologie des émotions, l'approche cognitive se donne les moyens non seulement de vérifier ses propres hypothèses, mais aussi de raffiner ses positions. Si bien qu'elle permet d'atteindre à la compréhension de ces aspects d'un film qu'une approche historique, par exemple, n'explique que partiellement et de cerner au plus près le fonctionnement de l'esprit humain. En effet, toute explication concernant les effets signifiants d'un film en particulier, dans la mesure où elle ne s'ancre exclusivement ni dans les réponses d'un spectateur ni dans les intentions d'un cinéaste, mène nécessairement à formuler des observations générales quant à la manière dont notre esprit fonctionne, observations qui, si elles valent pour un film en particulier, doivent également valoir pour d'autres films dotés des mêmes qualités.

Ce qui est versé au compte d'un troisième sens du film, ces aspects d'un film qui sont dits en excès, on gagnera à les concevoir comme des réponses de nature émotionnelle. Cela permettra d'abord de sortir le style de cette ornière que constituent les objectifs généralement assignés au film : dénoter, connoter, narrer. On dira donc que le troisième sens et l'excès transcendent ces buts immédiats, le but ultime du style étant de procurer une expérience. Nous gagnerons ainsi en intégration conceptuelle et en simplicité. Deuxièmement, il deviendra possible de fournir une explication causale des expériences émotionnelles vécues dans la rencontre avec un film, particulièrement celles qui débordent le cadre des émotions: selon le psychologue hollandais Nico Frijda (1993), "a monitoring of concerns" est à l'origine des émotions, par quoi nous sommes en prise sur notre environnement. Et c'est quand nous sommes plongés dans des situations dont les enjeux nous échappent que surgissent les émotions non définies par la cognition. Enfin, en considérant les émotions non définies par la cognition - être ému, être emporté — comme provenant du même système émotionnel 
que les émotions bien définies — la colère ou la peur ressenties dans des situations où les enjeux sont clairs - , nous éviterons toute "subjectivisation" ou "objectivisation" injustifiée des expériences vécues dans la rencontre avec un film. L'alternative même se trouve récusée puisque une réponse émotionnelle celle qui est définie par la cognition comme celle qui ne l'est pas - s'ancre tout autant dans les structures sémantiques du film que dans le système émotionnel du spectateur.

\section{Le concept de style}

Quand Barthes cherche à définir le troisième sens et Thompson, l'excès, tous deux accordent une importance certaine à la question du style. Que notre propre état émotionnel surgisse en réponse à des traits stylistiques du film, cela est évident pour Barthes (1982, p. 55), et c'est pourquoi il précise que le troisième sens, ce "signifiant sans signifié", se trouve "en dehors du langage (articulé), mais cependant à l'intérieur de l'interlocution ». Chez Thompson (1999, p. 496 et 499), le concept même de style est explicite. En effet, il est partie prenante de sa définition de l'excès: le style est, en combinaison avec la forme, un système unifié de structures caractéristiques de l'œuvre, l'excès recueillant ces aspects matériels qui ne forment aucun motif caractéristique.

Seulement, leur conception trop étroite du style empêche Barthes et Thompson de développer davantage les concepts de troisième sens et d'excès. Contemplant un photogramme d'Ivan le Terrible, Barthes $(1982$, p. 44) dit qu'il a beau voir les «[...] accidents signifiants [...] dont ce signe est composé », il n'arrive pas à nommer son signifié, ce qui le mène à affirmer que ce signe est "dès lors incomplet». Thompson (1999, p. 490) quant à elle fait simplement remarquer que quelquefois " [a] device has no function beyond offering itself for perceptual play ». En fait, il semble que les deux théoriciens pointent ce qui, d'un film, excède des objectifs qu'eux-mêmes assignent implicitement aux films: fournir du sens, raconter une histoire. Or, je l'ai dit en introduction, ce ne sont là que des buts immédiats, le but ultime étant de procurer une expérience. Je pense pour ma part être en mesure d'expliquer pourquoi un certain type d'état émo- 
tif émerge du film Ivan le Terrible. Mais pour ce faire, il faut caractériser le style de manière plus large, de sorte que le concept inclue les buts immédiats et les buts ultimes. À cet effet, je suggère de comprendre le style à la lumière de deux propositions.

La première peut s'énoncer comme suit: Parler de style, c'est nécessairement présupposer un but. En d'autres termes, le concept de style ne s'applique qu'aux artefacts, aux produits du labeur humain. "What is the style of a tulip, or an alpine meadow, or a pristine lake in the high Sierras?» demande Kendall Walton (1987, p. 73). Pour lui, la notion de style ne saurait s'appliquer aux objets naturels, aussi magnifiques soient-ils et aussi "esthétique " que soit l'intérêt avec lequel on les contemple, parce que leur existence ne doit rien à un acte intentionnel. Mais but s'entend ici en deux sens: soit on examine la manière dont une œuv.re est faite, et on considère que le but implicite a été de lui donner une facture particulière la constituant comme un tout singulier, ce qui permet de l'attribuer; soit on s'attache à ce que l'œuvre fait, et on considère que le but implicite est cet effet qu'elle produit, lequel effet l'inscrit souvent dans une catégorie esthétique générale. Ainsi, on ne peut dire de Le Pré de Bejine qu'il est caractéristique du style d'Eisenstein puisque ce dernier ne l'a jamais monté, mais on peut parler du style impressionniste d'une photographie produisant une sensation fugitive, par exemple celle d'être étendu sous un soleil excessif ${ }^{3}$.

"Stylistic terms are either derived inductively or deductively" (Arnheim, 1986, p. 264). Cette seconde proposition attire notre attention sur les processus mentaux en jeu dans l'analyse du style: l'induction, processus ascendant (mode "bottom-up»), et la déduction, processus descendant (mode «top-down»). Ainsi, identifier les parties d'un film — le début, la fin — au regard de leur style ou repérer des motifs - une répétition, une variation —, ce sont là des opérations inductives. Mais lorsqu'on remarque que le jeu d'un acteur permet de faire l'expérience de la situation du personnage comme si elle était réelle ou qu'une photographie produit une impression, par exemple celle d'être étendu au soleil, il s'agit de déductions. Les termes stylistiques dérivés de déductions circulent aisément dans les médias car, 
comme le souligne Arnheim (1986, p. 264), "[...] the "a priori" elapsed of categories can be shown to derive from constant properties of the instrument and the human agent $\%$.

Examinons maintenant à la lumière de ces propositions le concept de style le plus développé en études cinématographiques, celui proposé par Kristin Thompson et David Bordwell, tenants d'une approche formaliste. Dans le manuel Film Art: An Introduction, Bordwell et Thompson (1997, p. 357) décomposent la forme au sens large, entendue comme un système dynamique de relations, en deux systèmes formels: la forme proprement dite, qui correspond à des modes d'organisation, c'est-à-dire aux structures narrative ou non narrative; le style, c'est-à-dire l'usage qui est fait des techniques de mise en scène, de cinématographie, de montage et du son. «Style, then, is that formal system of the film that organizes film techniques" (Bordwell et Thompson, 1997, p. 357). Dans cette perspective systémique, le style, c'est l'ensemble des traits caractéristiques d'une œuvre ou d'un groupe d'œuvres. Ces traits, que l'on dégage par opérations déductives, permettent d'attribuer l'œuvre. Selon Bordwell et Thompson, il arrive que des traits stylistiques vaillent en soi. Mais cela est dit au passage, tandis que l'ensemble de leur propos fait du style un moyen au service des modes d'organisation du film, et surtout de la structure narrative. C'est dire que, considérant l'œuvre du point de vue de ce qu'elle fait, le but qu'on peut lui prêter par opération déductive reste celui de narrer une histoire. Voilà précisément ce qui amène Thompson à suggérer que certains éléments d'un film sont en excès. Cependant, si nous posons que l'objectif ultime de l'œuvre est d'engager le spectateur, de lui procurer une expérience qui vaut en soi, la narration - ou l'histoire qu'elle rapporte - devient l'occasion d'une expérience parmi d'autres.

Quant à Barthes, ce troisième sens qu'il sent à l'œuvre dans Ivan le Terrible l'arrache non seulement aux rets du récit, mais encore et surtout à la pratique même du sens. Barthes comprend qu'il fait là l'expérience du filmique, expérience qui l'amène là où la linguistique n'est d'aucun secours. N'en reste pas moins que l'essai de Barthes nous permet de poser que la production de sens, s'agirait-il d'un feuilleté de sens, ne saurait constituer 
l'objectif ultime d'un film. On la considérera donc comme un effet possible, un effet parmi d'autres ${ }^{4}$, et cela, bien que cet effet mobilise souvent toute notre attention.

Pour résumer notre position, nous dirons que le style doit être compris en un sens très large: le style est un moyen parmi d'autres, un des moyens dont disposent les films pour procurer des expériences émotionnelles. Ainsi, on peut apprécier les implications morales d'un conflit dans l'ordre de l'histoire, l'inventivité qui se manifeste dans la facture du film, la portée informative d'un film historique, l'aspect subversif d'une forme aussi bien que sa beauté ou son unité.

\section{Émotions et structures sémantiques contextuelles}

Pour associer le style aux émotions non définies par la cognition, il me faut caractériser ces dernières, ce que je ferai en les opposant aux émotions bien définies. Posons d'abord que les émotions ou les états émotionnels surgissent en réponse à ce que le psychologue Nico Frijda (1993) appelle des «situational meaning structures». Ce qui distingue une émotion d'une autre, ce n'est pas l'état corporel ou l'activité physionomique qui leur sont associés. Si les émotions se distinguent les unes des autres, si elles sont ressenties dans leur différence qualitative, c'est en raison de la structure sémantique contextuelle - ce qui provoque l'émotion, ce vers qui ou vers quoi elle est dirigée, qui a subi un tort ou un préjudice, etc. - propre à chacune. Par exemple, haine et colère different en ce que la haine est causée par une personne alors que la colère est causée par un événement aux coordonnées spatiotemporelles définies, en conséquence de quoi la haine se révèle plus durable. Ou encore, honte et culpabilité different en ce que la première surgit dans une situation où l'on est soi-même juge et partie, alors qu'on éprouve la seconde pour avoir causé préjudice à autrui.

Il est maintenant plus aisé de voir pourquoi certains aspects d'un film sont versés au compte d'un troisième sens ou considérés en excès. C'est qu'ils donnent lieu à des expériences émotionnelles se situant hors du cadre d'une structure contextuelle définie. Ces expériences semblent donc posséder une qualité phénoménologique unique. En présumant que les situations que 
Barthes et Thompson décrivent sont signifiantes en elles-mêmes - et je crois qu'elles le sont à cause des événements de l'histoire -, l'expérience du film acquiert une qualité distincte grâce aux détails vers lesquels notre attention est portée. Décrivant la scène d'ouverture d'Ivan le Terrible, alors qu'Ivan est couronné tsar, Barthes (1982, p. 44) décrit le maquillage des courtisans, il note le nez "bête» de l'un d'eux, le sourcil finement tracé de l'autre, éléments qui ne font pas simplement avancer le récit et qui ne fonctionnent pas symboliquement non plus. C'est que la caméra demeure assez longtemps sur les personnages pour nous faire découvrir ces caractéristiques, notre attention étant ainsi détournée des implications émotives de la scène. Pourtant, si ce n'était de la structure sémantique contextuelle sous-jacente - le couronnement d'Ivan est un moment très important de sa vie - nous ne pourrions peut-être pas apprécier les nombreux détails excessifs.

Le concept de structure sémantique de Frijda présuppose que l'individu est en contact avec son environnement: considérant une situation, il a l'impression que quelque chose est en jeu. Évaluant la situation, c'est du coup ses propres possibilités d'action qu'il évalue. Il faut alors évoquer "an action tendency": en colère, nous voulons blesser l'autre; apeurés, nous voulons nous enfuir ou nous cacher. Signalons au passage un développement utile pour la compréhension du jeu d'acteur et de l'expressivité d'un film. Pour Frijda (1993,p. 60 et 66), ce ne serait pas tant une forme spécifique et reconnaissable que nous détecterions, mais une fonction: l'expressivité servirait alors cette fonction en reliant le sujet à son environnement. Ainsi, un sourire ou un comportement très attentionné apparaissent comme des expressions de la peur dans telle situation dont nous avons déjà une certaine connaissance.

Quant au concept de tendance à l'action, il permet de comprendre pourquoi certaines scènes d'un film sont perçues comme réalistes. Le suspense fournit un bon exemple de ce que Ed Tan a nommé une tendance à l'action virtuelle: nous voudrions avertir la personne en danger, nous sommes même sur le point de crier un avertissement, nous souhaiterions intervenir. Même si elle est fictive, la situation est ressentie comme étant 
réelle. On remarquera que je n'oppose pas ici les émotions se rattachant au monde narré à celles se rattachant au film en tant qu'œuvre d'art, distinction que fait Ed Tan's. Une expérience sentie comme réaliste peut très bien avoir l'acteur, et non le personnage, comme cadre de référence. Je crois que c'est le cas, par exemple, avec le film Martha de Rainer-W. Fassbinder (1972) : par moments, le spectateur se sent pratiquement embarrassé pour la personne comme telle, et il apparaît secondaire que cette personne soit perçue en tant que Martha ou Margit Carstensen.

Considérant maintenant les états émotionnels non définis par la cognition, nous dirons avec Frijda (1993, p. 258) que ce sont «those modes of action readiness that manifest the fact that well-defined response cannot be developed: states of excitement, being disturbed, being moved, states of inhibition ". Ces états émotionnels se distinguent des émotions bien définies en ce qu'ils sont moins articulés: ils surgissent dans une situation dont nous n'arrivons pas à identifier les enjeux, une situation à laquelle nous ne savons pas très bien comment répondre, ni même s'il faut y répondre. Frijda (1993, p. 258) parle alors d'une "dedifferentiation of the emotion space". Lorsque Barthes (1982, p. 44) dit que le troisième sens n'imite rien, qu'il "excède la copie du motif référentiel ", il faut peut-être avancer une explication d'ordre psychologique: la situation ne présenterait pas d'enjeux offrant une prise à notre action, et ce, en raison d'une "dedifferentiation" de la signification contextuelle (Frijda, 1993, p. 56).

\section{L'inhibition en réponse à la scène finale d'Ivan le Terrible}

Les détails en excès, si manifestes dans Ivan le Terrible, ces aspects qui témoignent d'un troisième sens du film permettent de faire l'expérience du sublime, et ce, en raison de l'état d'inhibition dans lequel ils plongent le spectateur. Je commencerai par passer en revue les moyens mis en ouvre dans les scènes maîtresses du film pour offrir cette expérience du sublime, état émotionnel non défini par la cognition.

Notons d'abord que la séquence du couronnement comme la scène où le peuple russe rappelle son tsar à Moscou possèdent toutes deux une signification précise relevant de la situation. On 
$\mathrm{y}$ met en scène des moments extraordinaires de la vie du protagoniste, moments reposant sur des rites sociaux, sinon religieux, inhérents à la situation: tous se tiennent à l'écart d'Ivan ou bien, à la fin, s'agenouillent devant lui. La signification contextuelle de ces deux scènes se donne à nous de manière évidente, surtout celle de la scène finale, alors que nous avons été témoins des souffrances qu'ont apportées à Ivan ses efforts pour unifier la Russie.

Deuxièmement, et c'est là un élément important, notons que les détails excessifs ont une fonction: les intérêts en jeu dans le monde de l'histoire excluent que nous prenions part à l'action. À cet égard, les remarques de Torben Grodal ${ }^{6}$ sont particulièrement éclairantes. La distance entre l'avant-plan et l'arrière-plan dans la dernière scène contribue à évacuer le réalisme. L'accent est mis sur les mouvements d'Ivan quand il regarde aux cieux, mouvements amplifiés par la barbe fonctionnant comme un levier. La scène est ainsi empreinte d'une gravité qui n'offre aucune prise à la tendance à l'action du spectateur. Quant au cadrage, il coupe court aux processus d'anticipation: le spectateur n’est pas invité à prévoir la suite, il ne se demande pas comment Ivan réagira, s'il fera un discours ou pas. L'espace, dans cette scène finale, est tout simplement trop vaste pour être perçu comme une scène pour l'action. Et comme Ed Tan et Nico Frijda (1999, p. 62) l’ont suggéré, «[...] being in an environment in which one feels tiny and insignificant" inspire fondamentalement du respect.

Troisièmement, le jeu d'acteur revêt une importance décisive pour notre propos. Le jeu lent et retenu de Nicolai Cherkasov, ses postures impressionnantes, donnent à la scène une atmosphère de grandeur. Ce style de jeu, on pourrait le qualifier de jeu idéalisé. Il est redevable à cette approche ayant précédé le naturalisme et selon laquelle l'essence du personnage ou l'émotion devait être communiquée de manière claire par une expression ferme et marquée. Ce type de jeu qui, du personnage, recherche l'expression la plus représentative en éliminant tout ce qui risque de lui conférer un caractère ambigu, on pourrait le mettre en rapport avec la sculpture. Cela est particulièrement frappant lorsque Ivan se penche en avant et porte une main à 
son oreille pour marquer qu'il écoute le chant de son peuple, alors qu'il tend vers l'arrière l'autre main tenant un bâton. La posture lui confere alors le poids d'une lourde sculpture.

Ces trois aspects contribuent à créer une scène offrant la possibilité de faire l'expérience du sublime. Rappelons-nous qu'Eisenstein a réalisé Ivan le Terrible à un moment de sa vie où il voulait créer une synthèse des différentes formes d'expression au lieu de les faire se heurter. David Bordwell (1993, p. 182) exprime l'idée d'Eisenstein en ces termes: «A film's form achieves organic unity by building representations toward an emotion-laden "image" of the theme." C'est particulièrement vrai de la scène qui clôt Ivan le Terrible: le récit et les techniques cinématographiques s'unissent au jeu d'acteur pour donner le sentiment que la mission d'Ivan est accomplie - il a unifié la Russie, son peuple s'agenouille devant lui, d'où le sentiment du sublime.

Mais cette expérience émotionnelle passe par un état d'inhibition. Nous nous conduisons sensiblement de la même façon que si un bon ami se mariait; nous demeurons tranquillement assis en silence pour ne pas rompre le cours de la cérémonie, pour ne pas ruiner un moment important. Cette inhibition est due à la signification contextuelle: nous sentons l'importance de la situation pour Ivan, nous sentons que ces rites sociaux marquent un moment extraordinaire. Et parallèlement, comme le souligne Thompson, notre attention est attirée par des éléments formels : la courbe que forme le peuple russe, la posture de Cherkasov qui imite l'architecture de l'édifice à l'intérieur duquel il se trouve. Mais le point sur lequel j'insiste ici, c'est que ces éléments sont fonctionnels. Ils empêchent que la scène soit sentie de manière réaliste en réprimant toute velléité d'action de la part du spectateur. C'est parce que notre attention est détournée par tous ces autres aspects que nous ne nous engageons pas sur le plan émotif de façon sentimentale et mélodramatique, faisant plutôt l'expérience du sublime.

Il est essentiel de noter que le fait que des états émotifs ne soient pas définis par la cognition n’implique pas que la cognition n'y joue aucun rôle, mais simplement que la situation dont nous prenons conscience n'exige pas que nous nous y engagions. 
Le spectateur est en état d'inhibition. Par peur de gâcher le moment, il a la sensation qu'il doit « rester tranquille», qu'il doit se contenter de suivre du regard les gestes d'Ivan. Il ressent que le protagoniste a mérité cette célébration, ce couronnement par le peuple.

\section{Le sublime en art}

Je tenterai maintenant de relier cette explication du sublime comme état émotif d'inhibition à quelques conceptions développées en philosophie. Récemment, Cynthia Freeland a tenté d'appliquer aux films le concept de sublime de Kant. «Sublime films prompt our appreciative, awed reflection on how they use the film medium to disturb in artful ways $"$, affirme Freeland. A cela, ajoutons que l'expérience émotionnelle aussi émerge de la façon dont le film a été fait. Freeland (1999, p. 82) avance ensuite que $[[\ldots]$ such reflection is elevating because it includes a moral perspective" reposant sur "[the] cognition of how human artists can offer powerful moral reflections in their work». Chose importante, Freeland (1999, p. 83) restreint le sublime à une réaction secondaire: "It is crucial to the sublime that one somehow feels exaltation, elevation or pleasure about what one is overwhelmed by or fears. " Cette dernière affirmation est compatible avec l'analyse d'Ivan le Terrible proposée plus haut. Il se pourrait très bien que nous réagissions à notre propre réaction d'inhibition, celle-ci ayant partie liée avec la confusion résultant de l'incertitude de la relation entre notre état émotif et l'objet de notre attention.

Quant à la réflexion d'ordre moral que provoqueraient certains films, je crois que Freeland suppose chez le spectateur une trop grande conscience des moyens, du comment de l'expérience du sublime. Certes, les films qui l'intéressent se prêtent bien à cette perspective morale. Mais si nous supposions un instant que Barthes ait fait une telle expérience du sublime en voyant la scène du couronnement qui ouvre Ivan le Terrible, il faudrait conclure qu'il a mal fait son travail. Car au lieu de réfléchir sur les significations et la morale, il s'arrête aux courtisans, à la barbe d'Ivan et à d'autres détails insignifiants. Je crois plutôt que l'expérience du sublime $-s$ 'il s'agit bien de ce dont nous 
devons parler à propos d'Ivan le Terrible - est vécue directement et immédiatement, que c'est une expérience sensorielle, ainsi que l'entend Thompson.

Dans la foulée de Mary Mothersill, on préférera au sublime kantien la notion du sublime ébauchée par le rhéteur antique Longinus, la plus ancienne que l'on connaisse. Dans $O n$ the Sublime, un manuel de rhétorique, Longinus (1985, p. 8-9) vient près d'une définition lorsqu'il dit que $"[\ldots]$ sublimity is a kind of height and conspicuous excellence in speech and writing». Malheureusement, le propos est dans l'ensemble plutôt difficile à suivre, il est émaillé d'objections dirigées contre des contemporains, tandis que le sublime n'est traité qu'au passage. Néanmoins, le concept de "loftiness" apparaît consistant. Selon Mothersill (1995, p. 408), Longinus considère le sublime comme un style grandiose, "[...] suited only for subjects that are themselves lofty, magnificent and astonishing». Dans un passage où Longinus (1985, p. 41-42) aborde plus directement la question du sublime, il réfléchit sur l'application d'un style grandiose à un matériau capable de le supporter :

We must also look into what appears lofty in sections of poems and speeches and writings, to see whether it has just an outer image of greatness, with a lot of random decorations, but which when looked into is found to be puffed up, the kind of thing it is better to sense oneself superior to than to wonder at. You see, by true sublimity our soul somehow is both lifted up and taking on a kind of exultant resemblance -- filled with delight and great glory.

Pour Longinus, le sublime ravit, soulève l'âme, l'emplit d'un sentiment de gloire. Ce sont ces propriétés qu'ont prêtées au sublime les philosophes et les critiques du début du XVIII siècle. De sorte que, étudiant Milton, Joseph Addison a été amené à faire le premier la distinction entre l'ordonné, le symétrique ou le beau - et l'édifiant - ou le sublime. Mais Addison a aussi considéré que relevaient du sublime les montagnes, les mers déchaînées ou les falaises aussi bien que les châteaux en ruine. Pour Mothersill, il s'agit d'une erreur fondamentale, erreur tenant de l'oxymore et que Kant a institutionnalisée en 
reliant esthétique et éthique. Le concept du sublime, maintientelle, gagne à être conçu à la manière de Longinus (1985, p. 411): on ne peut attribuer un caractère sublime quaux choses relevant du style et aux sentiments.

Le propos de Longinus confirme mon analyse d'Ivan le Terrible à deux égards. Premièrement, il appuie l'idée que l'effet produit par la scène d'ouverture et la scène finale du film est conditionnel à une structure sémantique contextuelle sous-jacente. Nous perdrions probablement tout intérêt si les éléments relevant du troisième sens ou du style grandiose n'étaient pas supportés par une telle structure sémantique, car on n'y verrait alors qu'exagération. Deuxièmement, il est intéressant de noter que Longinus attribue un style grandiose aussi bien à un discours qu'à un texte écrit. Comme je l'ai déjà fait remarquer, Barthes et Thompson semblent tous deux conscients de la façon dont le film s'adresse à eux. Et comme je l'ai aussi suggéré, l'état d'inhibition du spectateur est dû pour une bonne part au jeu d'acteur idéalisé de Tcherkasof, jeu essentiellement rhétorique.

\section{Conclusion}

Il n'est nul besoin d'assumer que tout aspect de notre expérience s'inscrive d'abord comme sens ni de présumer que tout trait stylistique d'un film serve une fin narrative. Ainsi, ce qui pour Barthes relève d'un troisième sens du film, ce qui pour Thompson semble en excès, ces éléments non conceptuels d'un film ont ici été considérés comme des éléments assumant une fonction. À cet égard, la scène finale d'Ivan le Terrible est éloquente, qui procure au spectateur une expérience émotionnelle, qui lui permet de faire l'expérience du sublime. La notion d'émotion non définie par la cognition revêt donc une grande importance: l'activité émotionnelle ne se résume pas aux seules réponses émotives surgissant dans des situations bien définies, situations suffisamment déterminées pour appeler telle ou telle émotion en particulier.

M'appuyant sur les propos de Barthes et de Thompson, j'ai pour ma part insisté sur le fait que le jeu d'acteur, dans Ivan le Terrible, a beaucoup à voir avec les effets d'ordres cognitif et émotionnel produits par le film. Ce jeu, sans couper court aux 
considérations portant sur ce que les personnages font ou sentent, n'est pas réaliste; il se situe plutôt du côté de la rhétorique. Et pourtant, il engage le spectateur. J'ai montré comment le jeu de Cherkasov - la solennité de ses gestes et de ses postures incite le spectateur à la contemplation. C'est la signification contextuelle qui, sur le plan cognitif, fait réaliser au spectateur l'importance du moment. Et comme la situation ne présente pas d'enjeu particulier, elle n'offre aucune prise à l'action: ce moment auquel le spectateur assiste n'appelle aucune participation de sa part. Surr le plan émotif se manifeste donc une certaine ambiguïté: la tendance à l'action est bridée, elle fait place à une espèce de "dedifferentiation" des réactions émotives qui pourraient lier le spectateur au monde de l'histoire; ce sont des émotions diffuses et contenues qu'éprouve le spectateur, rendu d'autant plus conscient des détails qu'il est en état d'inhibition. Si bien que, sur le plan phénoménologique, il est transporté, il ressent un sentiment d'élévation.

C'est peut-être l'occasion ici de revenir à Barthes, à cette idée récurrente chez lui de déguisement raté: ce qui de certains photogrammes le happe, c'est quelque chose de dérisoire, par exemple dans une certaine expression de la douleur. Mais le dérisoire ne tient pas à l'expression; il tient à la douleur même. Le dérisoire tel que perçu et entendu par Barthes, c'est ce qui nous éloigne du réalisme, "du "réel" comme nature, instance réaliste» (1982, p. 55). Le dérisoire évacue le cliché de la représentation de la douleur pour incarner une douleur jusque-là inconnue. Voilà qui nous amène à penser les réponses de Barthes et les nôtres en termes de sympathie envers les personnages, qui ne sont justement plus des personnages mais des êtres proposant une expérience réelle, expérience du sublime qui laisse indifférents nos schèmes sensori-moteurs, mais qui intéressent l'âme dans ce qu'elle a de plus vital. C'est peut-être cette même relation au film qui se cache au coeur de la notion d'excès développée par Thompson.

On objectera peut-être que je me suis fort éloigné du style, adoptant plutôt une approche holistique des personnages, mêlant les propriétés de l'histoire et l'analyse du jeu. Mais il faudra alors noter qu'on ne peut se contenter d'attribuer au style les 
détails retenant notre attention et d'attribuer la signification contextuelle à la narration. Parce que sous une telle conception court l'idée que l'histoire existerait indépendamment de sa réalisation perceptuelle, cette dernière devant être prise en charge par l'analyse stylistique. Comme je l'ai dit, une telle conception du style, qui pose l'indépendance du contenu et de la forme, permet certes l'identification de traits caractéristiques; mais elle ne fournit aucune explication, elle ne mène pas à saisir ce que fait le style, ce à quoi il vise. La distinction entre histoire et narration, entre énoncé et énonciation, n’est qu'un modèle qui permet certes de saisir quelques-unes des propriétés d'un film, mais pas toutes loin s'en faut. Lorsqu'on considère un jeu d'acteur non réaliste, par exemple, cette distinction se révèle inutile.

Au cœur de mon analyse de la scène finale d'Ivan le Terrible en tant qu'elle permet de faire l'expérience du sublime se trouve une distinction entre être ému comme résultant d'un processus inconscient de structures signifiantes et être conscient de détails qui ne semblent pas influer sur notre état émotif. L'une des fonctions générales de la conscience, comme l'a suggéré le neurologue Antonio Damisio (1999, p. 37), serait de lier les processus émotifs internes à la perception du monde extérieur. Ainsi, on peut avancer l'hypothèse que l'expérience du sublime est secondaire: cette expérience serait une réponse à notre propre état émotionnel, induit par les structures sémantiques contextuelles, et à notre état de conscience, cette dernière se portant sur ces détails qui semblent excessifs. C’est, plus précisément, le hiatus ou le saut entre un état émotionnel et un contenu de connaissance qui susciterait l'expérience du sublime. Cette dernière fonctionnerait alors à la manière de la métaphore, telle que l'ont théorisée George Lakoff et Mark Johnson (1999, p. $268-$ 269) : me sentant ému, transporté, soulevé, je ferais l'expérience d'un Je indépendant du Moi corporel, et cela, en raison des mouvements sensori-moteurs que provoque la métaphore.

Traduit de l'anglais par Sébastien Babeux Texte français établi avec le concours de Bernard Perron

Université de Copenhague 


\section{NOTES}

Une version préliminaire de cet article a fait l'objet d'une communication dans le cadre du symposium sur les "Problems of Representation in a Cognitive Theory of Representation ", tenu en mai 2001 à l'Université de Pécs, en Hongrie. Je remercie les participants pour leurs commentaires fort constructifs.

1. Dans la première partie de son article, Thompson (1999) développe une conception de l'excès qui s'éloigne de celle proposée par des tenants de l'approche poststructuraliste, par Stephen Heath notamment. Dans une perspective psychoanalytique, Heath identifie l'excès à ces éléments hétérogènes que la narration n'arrive pas à mettre en échec, qui reviennent tel le refoulé. Voir Heath (1985, p. 519). Je m’en tiendrai quant à moi à la proposition de Thompson.

2. Voir en particulier Tan (1996), Fasting (1996), Grodal (1997), Plantinga et Smith (1999).

3. Dans «Style», E. H. Gombrich (1968, p. 352) donne du style une définition très large qui inclut les deux buts que l'on peut prêter aut style: "Style is any distinctive, and therefore recognizable, way in which an act is performed or an artifact made or ought to be performed and made" .

4. Voir Bordwell (1996, p. 93).

5. Tan distingue les émotions basées sur la fiction des émotions basées sur les objets; la peur causée par le personnage (fiction) de l'émerveillement devant un spectacle (objets). Voir Tan, (1994, p. 13).

6. Mon approche differe toutefois de celle de Grodal. Se basant sur le modèle entrée/sortie, Grodal considère que la subjectivité résulte de conditions insuffisantes. Des processus cognitifs non conscients envoient alors un ordre: il faut empêcher toute action, il n'y a pas lieu d'agir. Quant à moi, je considère qu'il y a absence de tendance à l'action parce qu'aucune n'est survenue. Voir Grodal (2000, p. 101).

\section{RÉFÉRENCES BIBLIOGRAPHIQUES}

Arnheim, 1986: Rudolf Arnheim, "Style as a Gestalt Problem", dans New Essays on the Psychology of Art, Berkeley, University of California Press, 1986, p. 261-273.

Barthes, 1982: Roland Barthes, "Le troisième sens. Notes de recherche sur quelques photogrammes de S. M. Eisenstein", dans L'Obvie et l'obtus. Essais critiques III, France, Éditions du Seuil, 1982, p. 43-61.

Bordwell, 1985: David Bordwell, Narration in the Fiction Film, Madison, The University of Wisconsin Press, 1985.

Bordwell, 1993: David Bordwell, The Cinema of Eisenstein, Cambridge, Harvard University Press, 1993.

Bordwell, 1996: David Bordwell, "Convention, Construction, and Cinematic Vision ", dans David Bordwell et Noël Carroll (dir.), Post-Theory: Reconstructing Film Studies, Madison, The University of Wisconsin Press, 1996, p. 87-107.

Bordwell et Thompson, 1997 : David Bordwell et Kristin Thompson, Film Art: An Introduction, New York, The McGraw-Hill Companies, 1997.

Damasio, 1999: Antonio R. Damasio, The Feeling of What Happens: Body and Emotion in the Making of Consciousness, New York, Harcourt Brace, 1999.

Fasting, 1996: Barbara Fasting, Lea's Communication Series, Mahwah, Lawrence Erlbaum Ass., 1996.

Freeland, 1999: Cynthia A. Freeland, "The Sublime in Cinema", dans Carl Plantinga et Greg Smith (dir.), Passionate Views: Film, Cognition, and Emotion, Baltimore, John Hopkins Press, 1999.

L'expérience émotionnelle et le style 
Frijda, 1993: Nico H. Frijda, The Emotions, Cambridge et New York, Cambridge University Press, 1993.

Gombrich, 1968 : E. H. Gombrich, "Style", dans David Sills (dir.), The International Encyclopedia of the Social Sciences, New York, Free Press, 1968, p. 352-361.

Grodal, 1997: Torben K. Grodal, Moving Pictures: A New Theory of Film Genres, Feelings, and Cognition, Oxford, Clarendon Press, 1997.

Grodal, 2000: Torben K. Grodal, "Subjectivity, Objectivity and Aesthetic Feelings in Film», dans Ib Bondebjerg (dir.), Moving Images, Culture and the Mind, Luton, Luton University Press, 2000, p. 87-104.

Heath, 1985 : Stephen Heath, "Jaws, Ideology and Film Theory", dans Bill Nichols (dir.), Movies and Method, vol. 2, Los Angeles, University of California Press, 1985, p. 509-514.

Lakoff et Johnson, 1999: George Lakoff et Mark Johnson, Philosophy in the Flesh: The Embodied Mind and Its Challenge to Western Thought, New York, Basic Books, 1999.

Longinus, 1985 : Longinus, On the Sublime, James A. Arieti et John M. Crossett (traduction), New York, Edwin Mellen Press, 1985.

Mothersill, 1995 : Mary Mothersill, "Sublime", dans David Edward Cooper (dir.), A Companion to Aesthetics, Cambridge, Blackwell Reference, 1995.

Plantinga et Smith, 1999 : Carl Plantinga et Greg M. Smith (dir.), Passionate Views: Film, Cognition, and Emotion, Baltimore, John Hopkins University Press, 1999.

Tan, 1996: Ed Tan, Emotion and the Structure of Narrative Film: Film as an Emotion Machine, Mahwah, Lawrence Erlbaum Ass., 1996.

Tan, 1994: Ed Tan, "Film-Induced Affect as Witness Emotion", Poetics, n ${ }^{\circ} 23$, 1994, p. 7-32.

Tan et Frijda, 1999: Ed Tan et Nico Frijda, «Sentiment in Film Viewing», dans Carl Plantinga et Greg Smith (dir.), Passionate Views: Film, Cognition, and Emotion, Baltimore, John Hopkins Press, 1999, p. 48-64.

Thompson, 1999: Kristin Thompson, "The Concept of Cinematic Excess", dans Film Theory and Criticism: Introductory Readings, Oxford, Oxford University Press, 1999 , p. $487-498$.

Walton, 1987: Kendall L. Walton, "Style and the Products and Processes of Art", dans Berel Lang (dir.), The Concept of Style, Ithaca, Cornell University Press, 1987, p. $72-103$. 